\title{
ANÁliSE da QUALIDADE dAS ÁGUAS DAS PRECIPITAÇÕES EM ABERTO E EFETIVA EM UM FRAGMENTO SECUNDÁRIO DA MATA ATLÂNTICA, NO MUNICÍPIO DE VIÇOSA, MG ${ }^{1}$
}

\author{
Vinícius Valiati de Souza ${ }^{2}$, Herly Carlos Teixeira Dias³ ${ }^{3}$ Adriana Albuquerque da Costa² e José Carlos de \\ Oliveira Júnior ${ }^{4}$
}

\begin{abstract}
RESUMO - Este trabalho foi conduzido na Estação de Treinamento e Educação Ambiental Mata do Paraíso, localizada no Município de Viçosa, MG, e teve por objetivo analisar a qualidade das águas da precipitação atmosférica em aberto (PA) e da precipitação efetiva (PE) de um trecho mais recente de regeneração da mata natural secundária, no período compreendido entre março e outubro de 2004. Para isso foram demarcadas três parcelas de precipitação efetiva dentro da Mata do Paraíso, de onde foram retiradas as alíquotas para as análises físicas das águas da precipitação interna (PI) e do escoamento pelo tronco (Et). Os valores médios de pH da água da PA, PI e Et foram 6,55; 6,9; e 6,34, respectivamente. A condutividade elétrica $\left(\mu \mathrm{S} \mathrm{cm}^{-1}\right)$ da água da PA, PI e Et apresentou valores médios iguais a 41,57; 100,5; e 66,36, respectivamente. A cor aparente da água da PA, PI e Et apresentou valores médios iguais a 6,09; 165; e 223,92 unidades de cor, respectivamente. A turbidez da PA, PI e Et apresentou valores médios iguais a 1,53; 2,72; e 1,94 UNT, respectivamente. A cobertura florestal da Mata do Paraíso influenciou as variáveis físicas da água de precipitação efetiva, principalmente nas chuvas que ocorreram logo após períodos de estiagem. A floresta, através da precipitação interna e do escoamento pelo tronco, regulou o $\mathrm{pH}$ e aumentou a condutividade elétrica, a cor e a turbidez da água da chuva, que atingiu os solos da floresta.
\end{abstract}

Palavras-chave: Precipitação em aberto, precipitação efetiva e qualidade física da água.

\section{NET THROUGHFALL QUALITY ASSESSMENT IN A SPONTANEOUS FOREST ECOSYSTEM (MATA ATLÂNTICA) IN VIÇOSA, MG, BRAZIL}

\begin{abstract}
The purpose of the present work was to evaluate water quality of rainfall (RF), throughfall $(T F)$ and stemflow (SF) in a spontaneous forest ecosystem between March and October 2004. Three plots were established under the forest canopy where physical water quality analysis were carried out for throughfall and stemflow samples. Volume-weighted mean (VWM) values founded for $\mathrm{pH}$, Electrical Conductivity (EC) $\left(\mu \mathrm{cm}^{-1}\right)$, Dissolved Solids $(D S)$ and Turbidity (TB) were 6.55, 6.90 and 6.34; 41.57, 100.50 and 66.36; $6.09,165$ and 223.92; 1.53, 2.72 and 1.94 respectively to $R F, T F$ and $S F$. Rainfall physical water quality characteristics were affected by the canopy, specially after drought periods between storms. Net throughfall and stemflow had the capacity to regularize $R F p H$, besides significantly increasing EC, DS and TB. The study was carried out on the Mata do Paraíso (EPTEA-MP), research station of the Universidade Federal de Viçosa (UFV) in the city of Viçosa, Minas Gerais - Brazil.
\end{abstract}

Keywords: Rainfall, net throughfall and rainwater physical quality.

\footnotetext{
${ }^{1}$ Recebido em 03.07.2006 e aceito para publicação em 29.03.2007

${ }^{2}$ Estudantes de Engenharia Florestal da UFV. E-mail: <viniciusvaliati@yahoo.com.br>; <drialbuquerque@yahoo.com.br>. ${ }_{3}^{3}$ Departamento de Engenharia Florestal da Universidade Federal de Viçosa (UFV). E-mail: <herly@ufv.br >.

${ }^{4}$ Programa de Pós-Graduação em Ciência Florestal da UFV. E-mail: <oliveirajr_ufv@yahoo.com.br>.
} 


\section{INTRODUÇÃO}

As partículas sólidas em suspensão na atmosfera responsáveis pelas características da água de chuva de uma região geralmente são semelhantes aos constituintes do solo presentes na bacia hidrográfica, podendo sofrer interferência de outras fontes naturais (i.e. oceanos, vulcões e desertos) ou antrópicas (i.e. estradas, carvoarias e centros industriais).

A liberação de compostos ácidos derivados da oxidação de combustíveis ricos em compostos de carbono, enxofre e nitrogênio (i.e. combustíveis fósseis e combustão da biomassa) na atmosfera induz a precipitação de ácidos fortes sobre a superfície terrestre, alterando a composição físico-química das águas superficiais (STONER, 1984; MARTINELLI et al., 2003), dos solos e seus compartimentos (BINI e BERSOLIN, 1998; PETRONIO et al., 2001; MARTINELLI et al., 2003), e mudanças na composição florística das áreas atingidas. A água da chuva, quando interage com a vegetação, fica alterada quanto à sua constituição físico-química, aumentando a concentração de sais (PARKER, 1983; LLOYD e MARQUES FILHO, 1988; PRAKASA RAO et al., 1995; CORNU et al., 1998; HÖLSCHER et al., 2003).

A água da chuva, após entrar em contato com o dossel da floresta, tem suas características físico-químicas alteradas pela lixiviação de metabólitos dos tecidos das folhas, troncos e ramos e também pela lavagem de partículas provenientes da deposição seca que acumulam após o período de estiagem, sendo que as espécies folhosas sofrem maior lixiviação do que as coníferas (OKI, 2002).

O estudo dos efeitos da formação florestal na qualidade das águas das chuvas necessita de acompanhamentos periódico e prolongado. A determinação de parâmetros físico-químicos pode auxiliar as tomadas de decisão quanto ao manejo a ser adotado em uma bacia hidrográfica, visto que a qualidade da água da precipitação pode influenciar a qualidade dos ecossistemas.

Assim, este trabalho objetivou analisar a qualidade da água da precipitação atmosférica e da precipitação efetiva incidente em um fragmento secundário de Mata Atlântica, por meio de variáveis físico-químicas.

\section{MATERIAL E MÉTODOS}

\section{1. Área de estudo}

Este estudo foi realizado na Estação de Pesquisa, Treinamento e Educação Ambiental na Mata do Paraíso (EPTEA-MP), situada no Município de Viçosa, MG, nas coordenadas geográficas de $20^{\circ} 45^{\prime}$ de latitude sul e $42^{\circ} 51^{\prime}$ de longitude oeste, com área de aproximadamente 194 ha.

Segundo a classificação de Köppen, o clima da região é do tipo $\mathrm{Cwb}$ (mesotérmico com verões quentes e chuvosos e invernos frios e secos), apresentando temperaturas médias anuais de $21,8^{\circ} \mathrm{C}$ e precipitação pluviométrica média anual de $1.350 \mathrm{~mm}$.

A região apresenta formação florestal estacional semidecidual tropical, em parte caducifólia (VELOSO, 1991). Conforme a espécie florestal, poderá haver variação na queda das folhas, de meados de maio até praticamente fins de outubro (CASTRO et al., 1983).

A maioria dos solos é de textura argilosa, sendo classificados como Latossolos Vermelho-Amarelos distróficos nas áreas com perfis convexos, Câmbicos nos topos, Argissolos nas áreas de perfis côncavos e nos terraços e, finalmente, Hidromórficos aluviais no leito maior dos cursos d'água (CORREA, 1984).

\subsection{Coleta de dados}

As medições da precipitação atmosférica em aberto (PA) da Mata do Paraíso foram obtidas a partir de dois pluviômetros com área de captação de $163 \mathrm{~cm}^{2}$. Com leituras realizadas no dia posterior a um evento de chuva.

Para a quantificação da precipitação efetiva (PE), foram demarcadas três parcelas de $20 \mathrm{~m}$ x $20 \mathrm{~m}$. Em cada parcela, a fim de medir a precipitação interna (PI), foram instalados 25 pluviômetros com área individual de captação de $78,24 \mathrm{~cm}^{2}$ e distanciados $5 \mathrm{~m}$ entre si. Para a obtenção das amostras para análise laboratorial foram retiradas alíquotas dos nove pluviômetros centrais de cada parcela (Figura 1). Com o objetivo de evitar a entrada de material grosseiro, foi instalado um sistema de retenção na abertura de cada pluviômetro utilizado para a obtenção das alíquotas.

A água de escoamento pelo tronco (Et) foi coletada utilizando-se coletores arbóreos constituídos por espuma expansiva de poliuretano. Este material possibilita maior eficiência na captação do fluido e menor interferência microbiológica por ser inerte, isto é, baixa reatividade bioquímica (BALIEIRO, 2001). As parcelas alocadas para o estudo da precipitação interna foram subdivididas em quatro quadrantes (subparcelas com 10 x 10 m). Um dos quadrantes foi aleatoriamente escolhido para a instalação dos coletores arbóreos, tendo como critério de inclusão os indivíduos com DAP > 5,0 cm (Figura 2). 


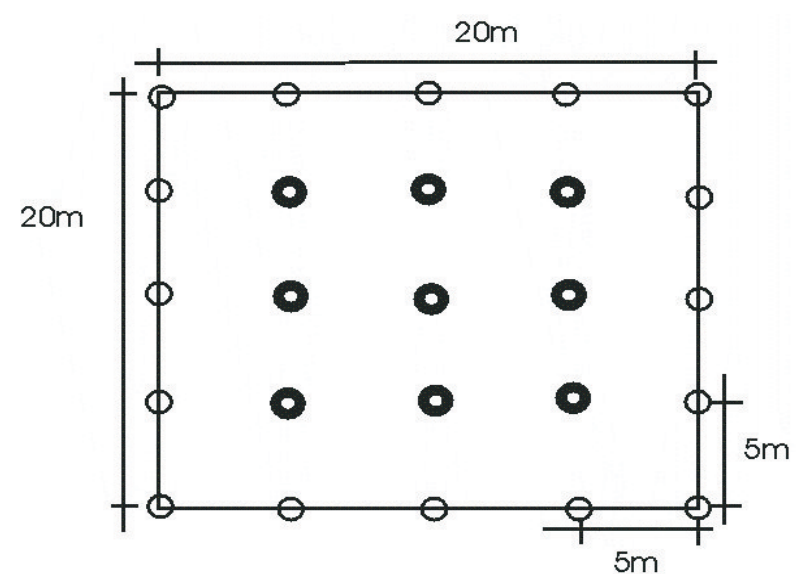

Figura 1 - Arranjo espacial dos pluviômetros nas parcelas para quantificação da precipitação interna. Em destaque, os pluviômetros utilizados para formarem a amostra composta utilizada nas análises laboratoriais - Mata do Paraíso, Viçosa, MG, 2003 - 2004.

Figure 1 - Throughfall sampling gauges arrangement. Gauges boldfaced were used to collect samples for laboratory analyses, Mata do Paraíso, Viçosa, MG, 2003-2004.

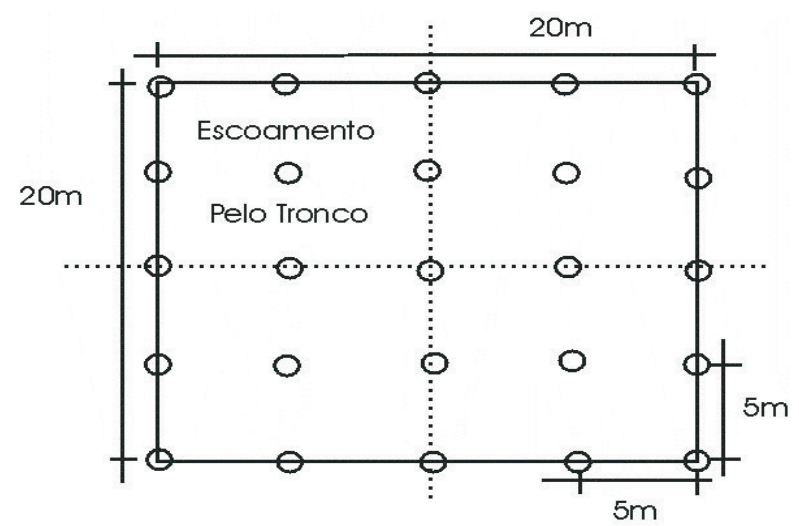

Figura 2 -Sistemática em quadrantes, utilizada para a instalação dos coletores arbóreos (escoamento pelo tronco), nos indivíduos com DAP $>5,0 \mathrm{~cm}$ - Mata do Paraíso, Viçosa, MG, 2003 - 2004.

Figure 2 -Stemflow collector placement on trees with DAP $>$ 5,0 cm, EPTEA-MP, Viçosa, MG, 2003-2004.

\subsection{Análises laboratoriais}

As amostras para análises laboratoriais foram acondicionadas em recipientes plásticos pretos (30 ml) e analisadas com no máximo 24 h após a coleta de campo no Laboratório da Estação de Tratamento de Água (ETA) situada no Campus da Universidade Federal de Viçosa (UFV).

A determinação do pH ocorreu com a utilização de pHmetro Digimed (DM 21), calibrado com soluçãopadrão 6,0 e 9,0. O valor referencial assumido neste estudo para classificar a chuva como sendo precipitação ácida ou natural foi o intervalo $\mathrm{pH}$ 5,5-5,6, resultante do equilíbrio entre o $\mathrm{CO}_{2}$ atmosférico e a precipitação, formando ácido carbônico (HAAG, 1985).

A condutividade elétrica foi determinada com a utilização de condutivímetro Digimed calibrado com solução-padrão $1.025 \mu \mathrm{S} * \mathrm{~cm}^{-1}$.

A coloração, cor aparente, das águas foi determinada pelo colorímetro Digimed, sendo a unidade de medida a Unidade Hazen ( $\mathrm{uH}$ ), ou apenas unidade de cor.

Para a determinação da turbidez foi utilizado turbidímetro Hach, modelo 2100 P, tendo como unidade a NTU.

Os dados foram armazenados em planilha apropriada, tabulados e submetidos a uma análise de correlação.

\section{RESULTADOS E DISCUSSÃO}

Os valores das análises (pH, $\mathrm{Ce}^{-}$, Cor e Turbidez) da água de chuva via precipitação em aberto, precipitação interna e escoamento pelo tronco e respectivos valores pluviométricos mensais, que ocorreram entre março e outubro de 2004 na Estação de Pesquisa, Treinamento e Educação Ambiental Mata do Paraíso, são apresentados no Quadro 1.

Pela Figura 3, percebe-se que os meses de março e abril, com precipitação média de $185,3 \mathrm{~mm}$, representam o final da estação chuvosa. Os meses de maio a agosto caracterizam o período de estiagem dessa região. No entanto, no ano em estudo observou-se que a estação de baixa pluviosidade estendeu-se até o mês de outubro. Isso pode ser um dos fatores que influenciaram as características físicas das águas de chuva analisadas, pois durante o período de baixa pluviosidade a tendência foi aumentar a concentração de partículas na atmosfera e a deposição nas copas e troncos da vegetação. Esse efeito da estacionalidade das chuvas nas características físicas das águas da precipitação efetiva (PE) pode ser visto claramente nos valores do Quadro 1. Esse resultado está de acordo com a observação de Levia e Frost (2003), em ampla revisão e avaliação da literatura disponível sobre o ciclo hidrológico e biogeoquímico de ecossistemas florestais e agrários, que indicam a sazonalidade como fator de interferência na qualidade da precipitação efetiva.

A precipitação em aberto (PA) apresentou, em media, água com $\mathrm{pH}=6,55$, com valor mínimo de $\mathrm{pH}=5,98$ e

R. Árvore, Viçosa-MG, v.31, n.4, p.737-743, 2007 
máximo de $\mathrm{pH}=7,32$. A precipitação interna (PI) apresentou, em média, água com $\mathrm{pH}=6,9$, sendo o valor mínimo de $\mathrm{pH}=6,49$ e máximo de $\mathrm{pH}=7,31$. A água coletada do escoamento pelo tronco apresentou, em média, $\mathrm{pH}=6,34$, com valor mínimo de $\mathrm{pH}=6,21$ e máximo de $\mathrm{pH}=6,53$. Esses resultados podem ser observados no Quadro 1. Apesar de os valores médios de $\mathrm{pH}$ da água da PA e PI serem similares, o desvio-padrão dos valores observados na PI $(\sigma= \pm 0,48)$ é menor quando comparados com os obtidos para a PA $(\sigma= \pm 0,8)$. Isso pode indicar menor capacidade de controle das condições de $\mathrm{pH}$ da água das chuvas via compartimento atmosférico, conferindo à vegetação local importante função no controle qualitativo da precipitação atmosférica que atinge o solo da floresta (CORNU et al., 1998).

Quadro 1 - Valores da precipitação em aberto (PA), precipitação interna (PI), escoamento pelo tronco (Et), condutividade elétrica $\left(\mathrm{Ce}^{-}\right)$, Cor, $\mathrm{pH}$ e turbidez, nos números de eventos analisados (n) entre março e outubro, observados na Mata do Paraíso, Viçosa, MG, 2004

Table $1-p H$, electrical conductivity, dissolved solids and turbidity values for rainfall ( $R F)$, throughfall (TF) and stemflow (SF) associated with the number of events analyzed from March to October in area of Mata do Paraíso, Viçosa-MG, 2004

\begin{tabular}{|c|c|c|c|c|c|c|}
\hline Mês & & $\mathrm{mm}$ & $\mathrm{PH}$ & $\begin{array}{c}\mathrm{Ce}^{-} \\
\left(\mu \mathrm{S} \mathrm{cm}^{-1}\right)^{-}\end{array}$ & Cor & Turbidez \\
\hline \multirow[t]{3}{*}{ Mar } & PA & 139,0 & 7,32 & 25,91 & 3,88 & 0,84 \\
\hline & PI & 108,7 & 7,18 & 133,28 & 107,38 & 1,44 \\
\hline & Et & 5,6 & 6,53 & 34,61 & 84,74 & 1,10 \\
\hline \multirow[t]{3}{*}{ Abr } & PA & 231,5 & 6,71 & 25,93 & 5,45 & 1,38 \\
\hline & PI & 193,3 & 6,75 & 41,15 & 61,39 & 1,62 \\
\hline & Et & 8,4 & 6,35 & 37,58 & 149,96 & 1,54 \\
\hline \multirow[t]{3}{*}{ Mai } & PA & 24,2 & 5,98 & 51,03 & 3,90 & 1,13 \\
\hline & PI & 18,5 & 7,31 & 99,53 & 33,12 & 1,43 \\
\hline & $\mathrm{Et}$ & 0,5 & 6,45 & 76,90 & 94,61 & 1,19 \\
\hline \multirow[t]{3}{*}{ Jun } & PA & 55,7 & 6,11 & 28,28 & 3,00 & 1,09 \\
\hline & PI & 48,2 & 7,00 & 61,69 & 118,13 & 2,19 \\
\hline & $\mathrm{Et}$ & 2,2 & 6,33 & 55,39 & 152,49 & 1,52 \\
\hline \multirow[t]{3}{*}{ Jul } & PA & 50,9 & 6,61 & - & 7,50 & 2,22 \\
\hline & PI & 39,9 & 6,70 & - & 259,74 & 2,79 \\
\hline & Et & 1,74 & 6,21 & - & 272,06 & 2,47 \\
\hline \multirow[t]{3}{*}{$\overline{\mathrm{Ago}}$} & PA & 0,0 & - & - & - & - \\
\hline & PI & 0,0 & - & - & - & - \\
\hline & Et & 0,0 & - & - & - & - \\
\hline \multirow[t]{3}{*}{ Set } & $\mathrm{PA}$ & 0,0 & - & - & - & - \\
\hline & PI & 0,0 & - & - & - & - \\
\hline & Et & 0,0 & - & - & - & - \\
\hline \multirow[t]{3}{*}{$\overline{\text { Out }}$} & PA & 23,6 & 6,58 & 153,79 & 6,32 & 4,65 \\
\hline & PI & 19,21 & 6,49 & 166,84 & 415,34 & 7,38 \\
\hline & Et & 0,58 & 6,22 & 127,31 & 589,67 & 3,83 \\
\hline
\end{tabular}

R. Árvore, Viçosa-MG, v.31, n.4, p.737-743, 2007

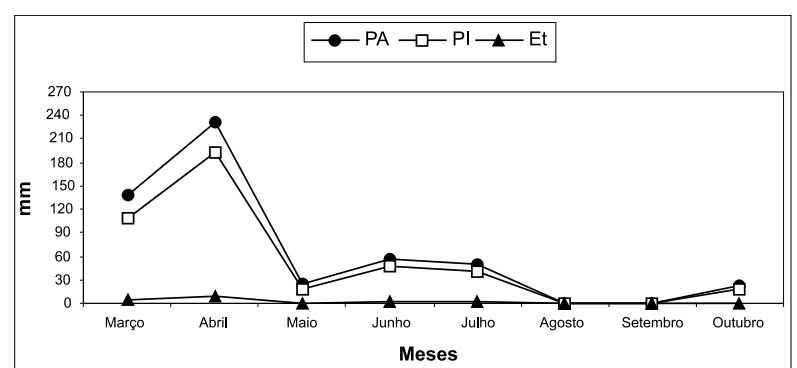

Figura 3 - Valores em mm da precipitação em aberto (PA), precipitação interna (PI ) e escoamento pelo tronco (Et) observados na Mata do Paraíso, Viçosa, MG, 2004.

Figure 3 - Rainfall $(R F)$, throughfall $(T F)$ and stemflow $(S F)$ values expressed in mm verified at Mata do Paraíso, Viçosa, MG, 2004.

A Figura 4 ilustra nitidamente esse efeito, sobretudo para os meses de março, abril e maio, quando o $\mathrm{pH}$ da água de PA variou de 5,98 - 7,32, enquanto os valores de $\mathrm{pH}$ da PI variaram numa faixa mais básica de 6,75 - 7,31. A dinâmica do pH da água de Et foi similar ao apresentado pela água da PI, porém em uma faixa um pouco mais ácido, influenciado pela lixiviação de compostos com características ácidas pelo fuste das espécies florestais. De forma geral, observa-se, pela Figura 4, que durante o período de estiagem o pH da água da precipitação em aberto diminui sensivelmente, enquanto o pH da precipitação interna se eleva, provavelmente pela quantidade de concentrados que permitem o aumento do $\mathrm{pH}$. Nesse período, o $\mathrm{pH}$ da água do escoamento pelo tronco permaneceu estável ou com pouca variação.

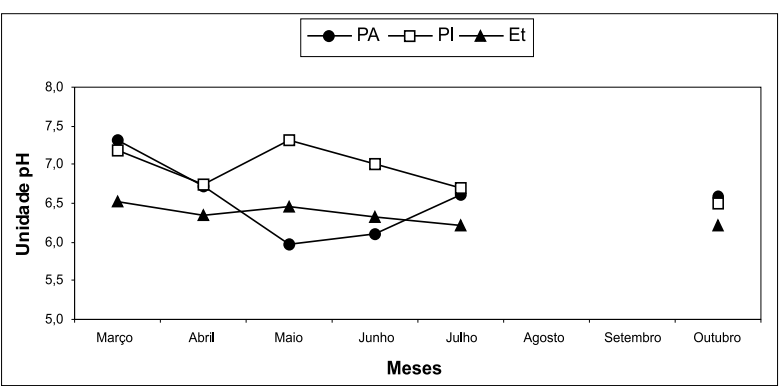

Figura 4 - Valores de pH da água de precipitação em aberto (PA), precipitação interna (PI) e escoamento pelo tronco (Et), observados na Mata do Paraíso, Viçosa, MG, 2004.

Figure 4-pH water for the rainfall (RF), throughfall (TF) and stemflow (SF) found in Mata do Paraíso, Viçosa, MG, 2004. 
A análise da água da precipitação pelo uso da variável capacidade condutiva da solução não permite distinguir quantitativamente o efeito de determinado nutriente ou composto. Contudo, esse parâmetro de análise qualitativo possibilita algumas inferências sobre a contribuição florestal na alteração da qualidade da água da PA, em comparação com a precipitação efetiva.

A condutividade elétrica média e respectivo desviopadrão, observados na água da PI $\left(100,5 \mu \mathrm{S} \mathrm{cm}^{-1}\right.$ e $\sigma$ $= \pm 51,25)$ foram maiores que os apresentados pela $\mathrm{PA}\left(41,57 \mu \mathrm{S} \mathrm{cm}^{-1}\right.$ e $\left.\sigma= \pm 22,3\right)$. Isso permite visualizar claramente o efeito da formação vegetal em estudo sobre a concentração de íons. O dossel da floresta tem a capacidade de retenção, via deposição gravitacional de particulados atmosféricos e liberação, por abrasão dos tecidos vegetais, de compostos, geralmente básicos, que elevam a concentração de íons da PA ao interagir com o dossel (JOHNSON e SWANK, 1973; OKI, 2002; PAIVA et al., 2002). A água do Et apresentou valores menores de condutividade elétrica do que aqueles exibidos pela água da PI e maiores do que para a PA na maioria dos eventos de chuva analisados; em média, a condutividade elétrica foi igual a $66,36 \mu \mathrm{S} \mathrm{cm}^{-1}(\sigma$ $= \pm 38,02)$ (Figura 5).

O aumento da condutividade elétrica indica estar ocorrendo, também, aumento na concentração de íons nesse compartimento durante os meses de abril e maio, o que está associado à diminuição na ocorrência de chuva nesse período. Os baixos valores da condutividade elétrica da água para a PA indicam que estas foram chuvas com poucos íons dissolvidos, que após a passagem pelo dossel recebeu adição de íons orgânicos e inorgânicos, tanto pelo compartimento da PI quanto do Et (LIU et al., 2004). Isso fica mais evidente quando se compara a concentração do período que vai de março a junho, com média de $83,91 \mu \mathrm{S} \mathrm{cm}^{-1}$, com a concentração de íons nas precipitações de outubro que representam as primeiras precipitações após o período de estiagem total, ou seja, após um período de acúmulo de particulados sobre o dossel da floresta.

A análise do parâmetro cor está associada à presença de compostos dissolvidos na solução, em sua maioria, de natureza orgânica (NETTO et al., 1987). As variações mensais da PA, PI e Et podem ser vistas na Figura 6. Observa-se que Et apresenta maiores valores de cor. isso provavelmente ocorreu pela maior capacidade de lixiviação de compostos orgânicos via escoamento pelo tronco.
Os baixos valores verificados de cor da água de PA (Quadro 1) indicam ser este pobre em compostos orgânicos, apresentando, em média, 6,09 $(\sigma= \pm 3,65)$. Conforme o Quadro 2, não foi verificado correlação entre cor da água de PA com a PI e Et, todavia a correlação entre o par PI e Et foi diretamente proporcional. A interação com a vegetação elevou, extraordinariamente, a composição dos compostos dissolvidos, em termos de média, 27,23 vezes. API apresentou, em média, $165(\sigma= \pm 145,1)$ unidades de cor. O Et lixiviou maior número de compostos, apresentando média de 223,92 $(\sigma= \pm 191,2)$.

A distribuição em maior amplitude, conforme desviopadrão, dos sólidos solúveis nos compartimentos PI e Et é esperada devido à presença de compostos orgânicos na água lixiviados dos tecidos vegetais. Os valores mais elevados desse parâmetro estão associados aos meses de julho $(259,74$ e 272,06$)$ e outubro $(415,34$ e 589,67), e cabe ressaltar que esses eventos de chuva ocorreram após um período de estiagem, o que proporcionou acúmulo de compostos solúveis no dossel e fuste arbóreos (Figura 6).

As águas da PA, PI e Et apresentaram médias 1,53 $(\sigma= \pm 0,67) ; 2,72(\sigma= \pm 2,03)$; e 1,94 $(\sigma= \pm 1,04)$ unidades de turbibez, respectivamente. Pela Figura 7 , nota-se que os compartimentos apresentaram curvas com traçados semelhantes, mas a presença de sólidos em suspensão nas amostras da PI foi sempre superior aos encontrados no Et e na PA. Isso confirma que a interceptação dos particulados atmosféricos pelo dossel da vegetação durante os meses sem precipitação pluvial aumenta. Essa tendência está associada à área de interceptação fornecida pelas folhas que compõem o dossel.

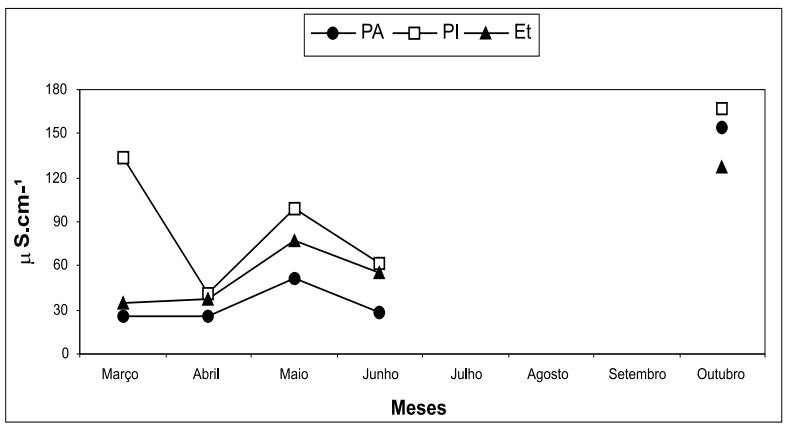

Figura 5-Condutividade elétrica $\left(\mathrm{mS} \mathrm{cm}^{-1}\right)$ da água de precipitação em aberto (PA), precipitação interna (PI ) e escoamento pelo tronco (Et), observados na Mata do Paraíso, Viçosa, MG, 2004.

Figure 5-Electrical conductivity water for the Rainfall (RF), throughfall (TF) and stemflow (SF) found for Mata do Paraíso, Viçosa, MG, 2004.

R. Árvore, Viçosa-MG, v.31, n.4, p.737-743, 2007 
Quadro 2 - Matriz de correlação entre as variáveis analisadas na Mata do Paraíso, Viçosa, MG, 2005

Table 2 - Correlation matrix for the analyzed variables in area of "Mata do Paraíso ", Viçosa, MG, 2005

\begin{tabular}{|c|c|c|c|c|c|c|c|c|c|c|c|c|c|c|c|c|}
\hline & & \multicolumn{3}{|c|}{$\mathrm{mm}$} & \multicolumn{3}{|c|}{$\mathrm{pH}$} & \multicolumn{3}{|c|}{ Conde } & \multicolumn{3}{|c|}{ Cor } & \multicolumn{3}{|c|}{ Turb } \\
\hline & & Patm & PI & ET & Patm & PI & ET & Patm & PI & ET & Patm & PI & ET & Patm & PI & ET \\
\hline \multirow[t]{3}{*}{$\overline{\mathrm{mm}}$} & Patm & 1,000 & & & & & & & & & & & & & & \\
\hline & PI & 0,999 & 1,000 & & & & & & & & & & & & & \\
\hline & ET & 0,995 & $\mathbf{0 , 9 9 8}$ & 1,000 & & & & & & & & & & & & \\
\hline \multirow[t]{3}{*}{$\overline{\mathrm{pH}}$} & Patm & 0,377 & 0,372 & 0,395 & 1,000 & & & & & & & & & & & \\
\hline & PI & 0,453 & 0,492 & 0,526 & 0,031 & 1,000 & & & & & & & & & & \\
\hline & ET & $-0,421$ & $-0,437$ & $-0,478$ & $-0,860$ & $-0,437$ & 1,000 & & & & & & & & & \\
\hline \multirow[t]{3}{*}{$\overline{\text { Cond }}$} & Patm & $-0,440$ & $-0,447$ & $-0,484$ & $-0,955$ & $-0,214$ & $\mathbf{0 , 9 5 3}$ & 1,000 & & & & & & & & \\
\hline & PI & 0,339 & 0,373 & 0,387 & $-0,471$ & $\mathbf{0 , 8 3 7}$ & 0,005 & 0,260 & 1,000 & & & & & & & \\
\hline & ET & $-0,368$ & $-0,384$ & $-0,430$ & $-\mathbf{0 , 8 8 5}$ & $-0,363$ & $\mathbf{0 , 9 8 1}$ & $\mathbf{0 , 9 7 7}$ & 0,125 & 1,000 & & & & & & \\
\hline \multirow[t]{3}{*}{ Cor } & Patm & 0,393 & 0,381 & 0,387 & 0,494 & $-0,183$ & $-0,522$ & $-0,739$ & $-0,051$ & $-0,675$ & 1,000 & & & & & \\
\hline & PI & $-0,422$ & $-0,433$ & $-0,438$ & $-0,204$ & $-0,506$ & 0,133 & 0,306 & $-0,007$ & 0,258 & 0,561 & 1,000 & & & & \\
\hline & ET & $-0,242$ & $-0,265$ & $-0,285$ & $-0,213$ & $-0,656$ & 0,264 & 0,279 & $-0,189$ & 0,357 & 0,623 & $\mathbf{0 , 9 3 0}$ & 1,000 & & & \\
\hline \multirow[t]{3}{*}{ Turb } & Patm & $-0,296$ & $-0,326$ & $-0,374$ & $-0,637$ & $-0,706$ & $\mathbf{0 , 8 3 2}$ & 0,796 & $-0,127$ & $\mathbf{0 , 8 8 9}$ & 0,004 & 0,531 & $\mathbf{0 , 7 0 7}$ & 1,000 & & \\
\hline & PI & $-0,368$ & $-0,380$ & $-0,383$ & $-0,218$ & $-0,516$ & 0,157 & 0,167 & $-0,029$ & 0,138 & 0,599 & 0,966 & $\mathbf{0 , 9 4 9}$ & 0,537 & 1,000 & \\
\hline & ET & $-0,322$ & $-0,337$ & $-0,322$ & 0,654 & $-0,550$ & $-0,453$ & $-0,692$ & $-0,814$ & $-0,634$ & 0,529 & 0,444 & 0,383 & $-0,123$ & 0,433 & 1,000 \\
\hline
\end{tabular}

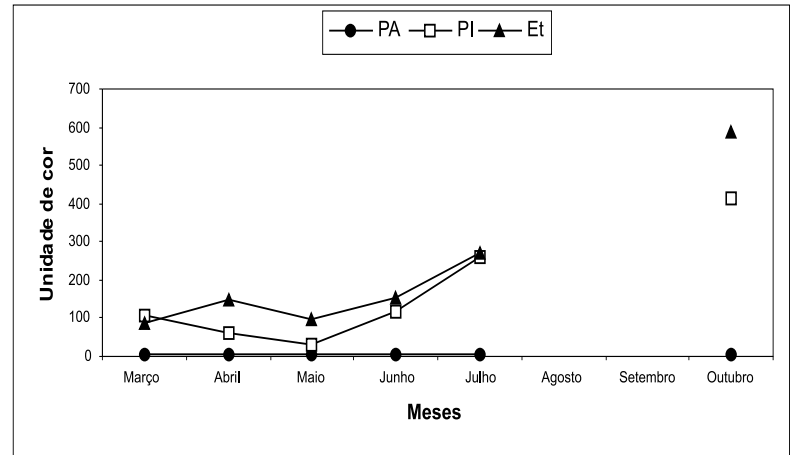

Figura 6 - Cor aparente observada para a precipitação em aberto (PA), precipitação interna (PI) e escoamento pelo tronco (Et), observados na Mata do Paraíso, Viçosa, MG, 2004.

Figure 6-Apparent color for the Rainfall (RF), throughfall (TF) and stemflow (SF) found in Mata do Paraíso, Viçosa, MG, 2004.

\section{CONCLUSÃO}

A cobertura florestal da Mata do Paraíso influenciou os valores dos parâmetros físicos da água de chuva, principalmente das chuvas que ocorreram logo após um período de estiagem. Afloresta através da precipitação interna, e do escoamento pelo tronco, regulou o $\mathrm{pH}$ e aumentou a condutividade elétrica e a cor e turbidez da água da chuva que atinge os solos da floresta.

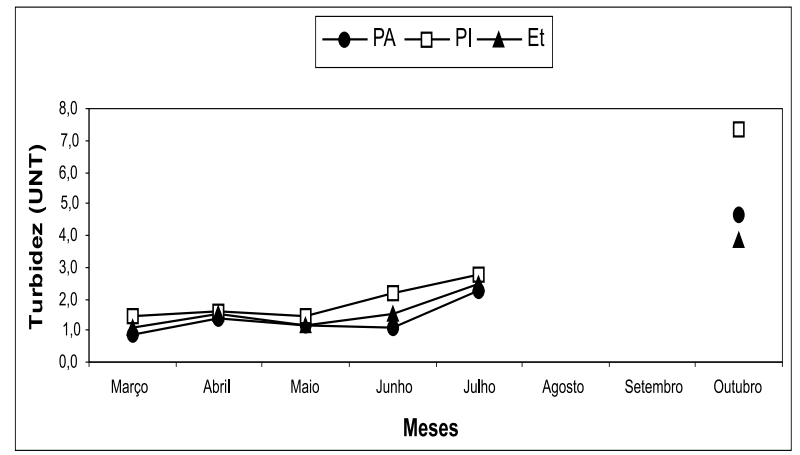

Figura 7 - Turbidez da água de precipitação em aberto (PA), precipitação interna (PI ) e escoamento pelo tronco (Et), observados na Mata do Paraíso, Viçosa, MG, 2004.

Figure 7 -Water turbidity for the rainfall $(R F)$, throughfall (TF) and stemflow (SF) found in Mata do Paraiso, Viçosa, MG, 2004.

De forma geral houve pouca diferença da acidez da chuva entre os compartimentos. Contudo, a precipitação antes de atingir o dossel florestal possui maior faixa de variação. A condutividade elétrica foi mais pronunciada na PI, indicando maior lixiviação de íons. O Et proporcionou as maiores taxas de compostos solúveis, indicadas pela cor. A maior taxa de turbidez ocorreu na água da PI, indicando o potencial do dossel da floresta para acumular particulados atmosféricos. 


\section{REFERÊNCIAS}

BALIEIRO, F. C. et al. Avaliação de um coletor de água de escoamento pelo tronco. Revista Árvore, v.25, n.2, p.167-173, 2001.

BINI, C.; BERSOLIN, F. Soil acidification by acid rain in forest ecosystems: A case study in northern Italy. The Science of the Total Environment, v.222, p.1-15, 1998.

CASTRO, P. S. et al. Interceptação da chuva por mata natural secundária na região de Viçosa-MG. Revista Árvore, v.7, n.1, p.76-89, 1983.

CORNU, S. et al. Origin and behaviour of dissolved chlorine and sodium in brazilian rainforest. Water Research, v.32, p.1151-1161, 1998.

CORREA, G. F. Modelo de evolução e mineralogia da fração argila de solos do planalto de Viçosa, MG. 1984. 87f. Dissertação (Mestrado em Solos) - Universidade Federal de Viçosa, Viçosa,MG, 1984.

HAAG, H. P. Chuvas ácidas. Campinas: Fundação Cargil, 1985. 77p.

HÖLSCHER, D. et al. Nutrient fluxes in stemflow and throughfall in three sucessional stages of a upper montane rain forest in Costa Rica.

Journal of Tropical Ecology, v.19, p.557-565, 2003.

JOHNSON, P.L.; SWANK, W.T. Studies of cations budgets in the southern Appalachians on four experimental watersheds with contrasting vegetation. Ecology, n.54, p. 70-80, 1973.

LEVIA Jr, D. F.; FROST E. E. A review and evaluation of stemflow literature in the hydrologic and biogeochemical cycles of forested and agricultural ecosystems. Journal of Hydrology, v. 274, p.1-29, 2003.

LIU, C. P.; WANG, M. C. M.; SHEU, B.H. Characterization of organic matter in rainfall, throughfall, stemflow, and streamwater from three subtropical forest ecosystems. Journal of Hydrology, v.289, p.275-285, 2004.
LLOYD, C. R.; MARQUES FILHO, A. O. Spatial variability of throughfall and stemflow measurements in amazonian rainforest. Agricultural and Forest Meteorology, v.42, p.63-73,1988.

MARTINELLI, L. A. et al. Acid rain and nitrogen deposition in a sub-tropical watershed (Piracicaba): ecosystem consequences. Environmental Pullution, v.121, p.389-399, 2003.

NETTO, J. M. A. Técnica de abastecimento e tratamento de água. 2.ed São Paulo: CETESB/ASCETESB, 1987.435p.

OKI, V. K. Impactos da colheita de Pinus taeda sobre o balanço hídrico, a qualidade da água e a ciclagem de nutrientes em microbacias. 2002. $85 \mathrm{f}$. Dissertação (Mestrado em Recursos Florestais) Escola Superior de Agricultura "Luiz de Queiróz", Piracicaba, 2002.

PAIVA, H. N. et al. Lixiviação de nutrientes pela água de chuva em um fragmento de Mata Atlântica em Viçosa, MG. In: REUNIÃO BRASILEIRADE MANEJO ECONSERVAÇÃO DO SOLO EDA ÁGUA, 14., 2002, Cuiabá. Anais Resumos Completos... Cuiabá: 2002. p.1-1.

PARKER, G. G. Throughfall and stemflow in the forest nutriente cicle. Advences in Ecological Resources, v.13, p.57-133, 1983.

PETRONIO, B. M. et al. Effects of acid rain on soil humic compounds. Talanta, v.54, p.837-846, 2001.

PRAKASA RAO, P. S. et al. Rain water and throughfall chemistry in the Silent Valley forest in South India. Atmosferic Environment, v.29, p.2025-2029, 1995.

STONER, J. H. The effects of acidification on the ecology of streams in te upper Tywi catchment in west wales. Environmental Pollution. v.35, p.125-157, 1984.

VELOSO, H. P.; RANGEL FILHO, A. L. R.; LIMA, J. C. A. Classificação da vegetação brasileira, adaptada a um sistema universal. Rio de Janeiro: Instituto Brasileiro de Geografia e Estatística. 1991. 123p.

R. Árvore, Viçosa-MG, v.31, n.4, p.737-743, 2007 
